\title{
Revealing excited states dynamics in cross-linked covalent hybrids of graphene and diketopyrrolopyrrole oligomers via ultrafast transient absorption spectroscopy
}

\author{
Aaron M. Ross,,$^{*}$ Meng Zheng, ${ }^{2}$ Michele Maggini, ${ }^{2}$ Fabio Marangi, ${ }^{1,3}$ Giulio Cerullo, ${ }^{1,3}$ Teresa Gatti, ${ }^{4}$ Francesco \\ Scotognella ${ }^{1,3}$ \\ ${ }^{1}$ Department of Physics, Politecnico di Milano, Piazza L. da Vinci 32, 20133 Milano, Italy \\ ${ }^{2}$ Department of Chemical Sciences, University of Padova, via Marzolo 1, 35131, Padova, Italy \\ ${ }^{3}$ Center for Nano Science and Technology@PoliMi, Istituto Italiano di Tecnologia (IIT), Via Giovanni Pascoli, 70/3, 20133, \\ Milan, Italy \\ ${ }^{4}$ Center for Materials Research and Institute of Physical Chemistry, Justus Liebig University Giessen, Heinrich Buff Ring 17 , \\ 35392, Giessen, Germany.
}

\begin{abstract}
We report on ultrafast dynamics in cross-linked covalent hybrids of graphene and diketopyrrolopyrrole (TDPP) oligomers. Comparisons between non-polymerizing (EXG-TDPP) and polymerizing (c-EXG-TDPP) hybrids show that charge/energy transfer occurs faster than $50 \mathrm{ps}$ in EXGTDPP, while the c-EXG-TDPP system shows quenching within $18 \mathrm{ps}$. The transient response of c-EXGTDPP is completely quenched ( $>99 \%$ ), while in EXG-TDPP full quenching occurs after 1.39 ns. Photocurrent studies of c-EXG-TDPP films show IPCE $>2 \%$, indicating ultrafast charge transfer between TDPP and graphene.
\end{abstract}

\section{Introduction}

One promising class of synthetic systems in which efficient energy/charge transfer may be achieved is graphene-based materials (GBMs). Graphene monolayers exhibit exquisitely high carrier mobilities, broadband absorption (2.3\% per monolayer), and ultrafast optical non-linearities such as saturable absorption [1].

The utility of graphene can be enhanced further via functionalization, either with nanoparticles or molecules, and via covalent or non-covalent linkages [2]. Sensitization of graphene via attachment of stronglyabsorbing chromophores may provide a new avenue for the development of cheap and non-toxic solar cells. Additionally, the presence of graphene, whether via covalent or non-covalent linkage, has been shown to significantly modify the electronic properties of chromophores [3].

Here we investigate the ultrafast dynamics of mechanically-exfoliated few-layer graphene and diketopyrrolopyrrole oligomers via ultrafast transient absorption spectroscopy with 70 fs time resolution. Two hybrids are studied in comparison to the isolated $\mathrm{Ph}_{2}$ TDPP chromophore, one of which (c-EXG-TDPP) cross-links graphene flakes via long polymeric backbone chains of TDPP, and the other of which (EXG-TDPP) does not polymerize (Figure 1). While quenching of the transient optical absorption is observed in both samples, the efficiency of this quenching process is shown to be much higher in c-EXG-TDPP compared to EXG-TDPP.

\section{Results}

We examined a recently developed cross-linked covalent hybrid between few-layer graphene flakes and bifunctional diphenyl-dithiophenedikethopyrrolopyrrole $\left(\mathrm{Ph}_{2} \mathrm{TDPP}\right)$ units [3,4]; the resulting cross-linked structure

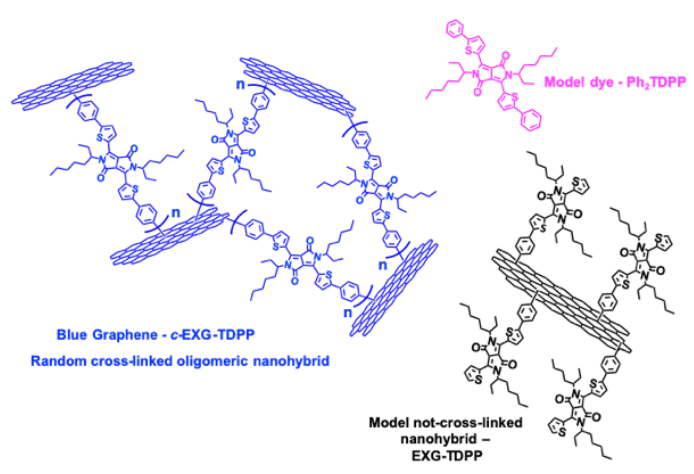

Figure 1: Structure of the cross-linked graphenedithiophenediketopyrrolopyrrole hybrid c-EXG-TDPP, of the model $\mathrm{Ph}_{2}$ TDPP chromophore unit constituting its cross-linking oligomers, and of the analogous hybrid system lacking cross-linking EXG-TDPP.

is referred to as c-EXG-TDPP. A similar model hybrid unit that does not cross-link is referred to EXG-TDPP (Figure 1). These samples are dissolved in toluene and placed in $1 \mathrm{~mm}$ path length cuvettes. It was confirmed here via steady-state linear absorption and emission experiments that the electronic properties of the crosslinked system c-EXG-TDPP are modified significantly compared to both $\mathrm{Ph}_{2}$ TDPP and EXG-TDPP: the main absorption band of $\mathrm{Ph}_{2} \mathrm{TDPP}$ is red-shifted by $66 \mathrm{~nm}$ in cEXG-TDPP, indicating a large increase in conjugation length along the polymeric backbone chain. These changes in electronic properties may explain the differences in charge/energy transfer processes between the two hybrids.

The ultrafast dynamics of both the base unit $\mathrm{Ph}_{2} \mathrm{TDPP}$, and the two hybrids, are studied via ultrafast transient absorption spectroscopy. A non-collinear optical parametric amplifier (NOPA) is pumped with $800 \mathrm{~nm}$,

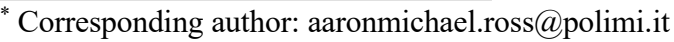




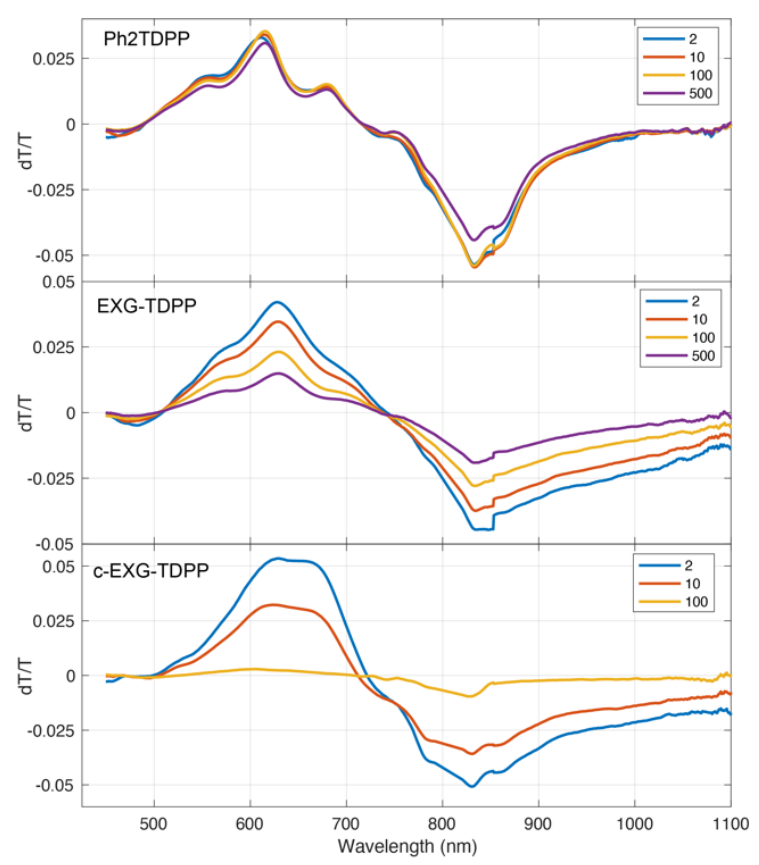

Figure 2: Transient absorption spectra for $\mathrm{Ph}_{2} \mathrm{TDPP}$, EXGTDPP, and c-EXG-TDPP at selected pump-probe delay times (labeled in picoseconds in legend). Discontinuity at $\sim 850 \mathrm{~nm}$ is due to stitching between different experimental runs.

150 fs pulses generated by a chirped pulse amplified (CPA) Ti:sapphire laser at a $1 \mathrm{kHz}$ repetition rate, yielding sub-100 fs pulses at 575, 625, and $645 \mathrm{~nm} \mathrm{[5],} \mathrm{used} \mathrm{to}$ pump $\mathrm{Ph}_{2}$ TDPP, EXG-TDPP, and c-EXG-TDPP, respectively. Broadband white light is generated in a sapphire crystal as a probe, and differential transmission $(\Delta \mathrm{T} / \mathrm{T})$ is measured by comparing the transmission of the probe through the sample when the pump is blocked/unblocked.

Global analysis of $\Delta \mathrm{T} / \mathrm{T}$ spectra (Fig. 2) using the sum of three exponentials allowed for the elucidation of energy/charge transfer processes. In EXG-TDPP, a process faster than $50 \mathrm{ps}$ is observed, followed by a much longer decay with a lifetime of $1.39 \mathrm{~ns}$, in agreement with previous TRPL measurements [3]. Ground state bleaching (GSB) features are observed at 575 and $645 \mathrm{~nm}$, stimulated emission (SE) signals are observed at $700 \mathrm{~nm}$; these results are in agreement with linear absorption and steady-state emission. A broad excited-state absorption (ESA) band is observed starting at $725 \mathrm{~nm}$, and extending out into the NIR past $950 \mathrm{~nm}$. The decay-associated spectrum (DAS) extracted via global fitting (not shown) associated with the 47 ps lifetime is broadened out into the NIR farther than both $\mathrm{Ph}_{2}$ TDPP ESA features and the longer EXG-TDPP DAS with lifetime of $1.39 \mathrm{~ns}$; this may indicate the presence of a charge-transfer state, which is followed by back electron transfer into the TDPP moiety.

However, much more efficient and rapid quenching is observed in c-EXG-TDPP: a broad DAS with lifetime $3.8 \mathrm{ps}$ is observed at longer wavelengths than the main GSB peaks, indicating an in-filling of the excited singlet states from higher-lying states. The full transient absorption signal is quenched within $18 \mathrm{ps}$. Since the GSB features observed from $600-660 \mathrm{~nm}$ in the $18 \mathrm{ps}$ DAS are also observed in linear absorption, and the ESA features are similar to those associated with the excited singlet states of $\mathrm{Ph}_{2}$ TDPP, we believe preliminarily that charge separation between the TDPP moieties and covalentlybound graphene occurs on the 18 ps timescale, and that the charge separated state may not be optically active. This phenomenon may be explained by the increased conjugation length in c-EXG-TDPP, which could lead to a more stable charge separated state than in EXG-TDPP. It is also expected that there is an interplay between charge transfer from nearest TDPP moieties attached directly to graphene, energy transfer to graphene from more distant TDPP moieties, and increased conjugation length in c-EXG-TDPP compared to oligomeric EXGTDPP.

Photocurrent measurements of c-EXG-TDPP films indicate that a charge transfer process is taking place, with incident photon to electron conversion efficiencies (IPCE) $>2 \%$. This result may confirm the rapid charge separation process occurring in c-EXG-TDPP; photocurrent collection is further enhanced likely due to transport of the extracted electron along the graphene scaffolding. Photocurrents were not detected for $\mathrm{Ph}_{2} \mathrm{TDPP}$ or nonfilm-forming EXG-TDPP drop-cast samples.

We conclude that energy/charge transfer processes in these hybrid graphene-dye systems that occur on sub 50 ps timescales are observed using ultrafast transient absorption spectroscopy, with charge transfer confirmed by photocurrent measurements.

\section{References}

1. D. R. Cooper, B. D'Anjou, N. Ghattamaneni, B. Harack, M. Hilke, A. Horth, N. Majlis, M. Massicotte, L. Vandsburger, E. Whiteway, V. Yu, ISRN Condens. Matter Phys. 2012, 501686 (2012).

2. G. Bottari, M. Ángeles Herranz, L. Wibmer, M. Volland, L. Rodríguez-Pérez, D. M. Guldi, A. Hirsch, N. Martín, F. D’Souza, T. Torres, Chem. Soc. Rev. 46, 4464 (2017).

3. M. Zheng, F. Lamberti, L. Franco, E. Collini, I. Fortunati, G. Bottaro, G. Daniel, R. Sorrentino, A. Minotto, A. Kukovecz, E. Menna, S. Silvestrini, C. Durante, F. Cacialli, G. Meneghesso, M. Maggini, T. Gatti, Synth. Met. 258, 116201 (2019).

4. P. E. Hartnett, E. A. Margulies, C. M. Mauck, S. A. Miller, Y. Wu, Y. L. Wu, T. J. Marks, M. R. Wasielewski, J. Phys. Chem. B 120, 1357 (2016).

5. C. Manzoni, D. Polli, and G. Cerullo, Rev. Sci. Instrum. 77, 1 (2006). 